\title{
Sensitivity to Small Delays of Pathwise Stability for Stochastic Retarded Evolution Equations
}

\author{
Kai Liu ${ }^{1,2}$
}

Received: 1 June 2016 / Revised: 11 December 2016 / Published online: 9 March 2017

(C) The Author(s) 2017. This article is published with open access at Springerlink.com

\begin{abstract}
In this paper, we shall study the almost sure pathwise exponential stability property for a class of stochastic functional differential equations with delays, possibly, in the highest-order derivative terms driven by multiplicative noise. Instead of establishing a moment exponential stability as the first step and then proceeding to investigate the pathwise stability of the system under consideration, we shall develop a direct approach for this problem. As a consequence, we can show that some systems, which are not exponential momently stable, have the exponential stability not sensitive to small delays in the almost sure sense.
\end{abstract}

Keywords Stability sensitive to small delays - Pathwise exponential stability · Stochastic delay evolution equation

Mathematics Subject Classification (2010) 60H15 · 60G15 · 60H05

The author is grateful to the Tianjin Thousand Talents Plan for its financial support.

$\bowtie \quad$ Kai Liu

k.liu@liverpool.ac.uk

1 College of Mathematical Sciences, Tianjin Normal University, Tianjin 300387,

People's Republic of China

2 Department of Mathematical Sciences, School of Physical Sciences, The University of Liverpool, Liverpool L69 7ZL, UK 


\section{Introduction}

We start with a simple one-dimensional linear differential equation

$$
\left\{\begin{array}{l}
\mathrm{d} y(t)=(\alpha+\beta) y(t) \mathrm{d} t, \quad t \geq 0, \\
y(0)=y_{0} \in \mathbb{R}
\end{array}\right.
$$

where $y(0)=y_{0} \in \mathbb{R}$, and $\alpha, \beta \in \mathbb{R}$. Since $y(t)=y_{0} e^{(\alpha+\beta) t}$ for all $t \geq 0$, it is easy to see that if $\alpha+\beta<0$, the null solution of (1.1) is exponentially stable.

Let $r>0$ and consider a time delay version of (1.1) in the form

$$
\mathrm{d} y(t)=\alpha y(t) \mathrm{d} t+\beta y(t-r) \mathrm{d} t, \quad t \geq 0 .
$$

Because of the time delay feature in (1.2), one need set up proper initial data different from those in (1.1) to make (1.2) well-defined. For instance, let $y(0)=\phi_{0} \in \mathbb{R}$ and $y(t)=\phi_{1}(t)$ for $t \in[-r, 0]$ where $\phi_{1} \in L^{2}([-r, 0], \mathbb{R})$. It is well known (see, e.g., [2] or [14]) that under the condition $\alpha+\beta<0$, there exists $r_{0}>0$ such that the null solution of (1.2) is exponentially stable for all $r \in\left(0, r_{0}\right)$, while for $r>r_{0}$, the null solution is exponentially unstable. In other words, exponential stability at this moment is not sensitive to small delays, a result which actually remains true in any finite dimensional context (see, e.g., Chap. 7 in [2]).

Now let us consider a linear one-dimensional Itô equation

$$
\mathrm{d} y(t)=(\alpha+\beta) y(t) \mathrm{d} t+\sigma y(t) \mathrm{d} w(t), \quad t \geq 0,
$$

where $w(t), t \geq 0$, is a standard real Brownian motion, $y(0)=y_{0} \in \mathbb{R}$, and $\alpha, \beta$ and $\sigma$ are real constants. A direct computation shows that the solution process $y(t), t \geq 0$, of (1.3) is explicitly given by

$$
y(t)=\exp \left\{\sigma w(t)+(\alpha+\beta) t-\sigma^{2} t / 2\right\} y_{0}, \quad t \geq 0 .
$$

Hence, by virtue of the well-known law of the iterated logarithm (cf. Revuz and Yor [13]), it is easy to see that the asymptotically exponential growth rate of solution $y$ is given by

$$
\limsup _{t \rightarrow \infty} \frac{\log |y(t)|}{t}=\alpha+\beta-\frac{\sigma^{2}}{2} \quad \text { a.s. }
$$

Therefore, the null solution of (1.3) has global exponential stability in the almost sure sense if and only if $\alpha+\beta<\sigma^{2} / 2$.

On the other hand, consider a time delay version of (1.3) in the following form

$$
\mathrm{d} y(t)=\alpha y(t) \mathrm{d} t+\beta y(t-r) \mathrm{d} t+\sigma y(t) \mathrm{d} w(t), \quad t \geq 0,
$$

where $r>0$. For pathwise stability of Eq.(1.6), it turns out that, in addition to the condition $\alpha+\beta<\sigma^{2} / 2$, the null solution of Eq. (1.6) could secure the same 
exponential stability in the almost sure sense as long as the delay parameter $r>0$ is sufficiently small. In fact, it is shown in Bierkens [3] that if $r>0$ is so small that

$$
|\beta| e^{3 \sigma^{2} r / 2}+\alpha<\sigma^{2} / 2
$$

then the null solution of (1.6) is exponentially stable almost surely. Thus, stability is not sensitive in this case to small delays. It is also worth mentioning that as a corollary of more general results for nonlinear stochastic systems, Appleby and Mao [1] showed that if $r>0$ is such a small number that

$$
2 e^{\sigma^{2} r}(|\beta|+\alpha) \Phi(\sigma \sqrt{r})<\sigma^{2} / 2
$$

then the null solution of (1.6) has the same pathwise exponential stability. Here $\Phi$ is the cumulative distribution function of a standard normal random variable.

In an infinite dimensional setting, the situation is quite different. In particular, it was observed by Datko et al. [6] (see also [5]) that small delays may destroy stability for a partial differential equation. More precisely, consider a deterministic differential equation in a Hilbert space $H$,

$$
\left\{\begin{array}{l}
\mathrm{d} y(t)=A y(t) \mathrm{d} t+B y(t-r) \mathrm{d} t, \quad t \geq 0, \\
y(0)=\phi_{0} \in H, \quad y(t)=\phi_{1}(t), \quad t \in[-r, 0], \phi_{1} \in L^{2}([-r, 0], H),
\end{array}\right.
$$

where $A$ generates a $C_{0}$-semigroup and $B$ is a linear operator in $H$. If the spectrum $\sigma(A)$ of $A$ is unbounded along an imaginary line, it was shown (see Theorem 7.4 in [2]) that for any $r_{0}>0$, one can always find an operator $B \in \mathscr{L}(H)$, the family of all bounded linear operators on $H$, and $r \in\left(0, r_{0}\right)$ such that $A+B$ generates an exponentially stable semigroup and meanwhile the system (1.7) is not exponentially stable. From this observation, we can see that the unboundedness of the spectrum set of $A$ along imaginary axes may cause trouble in the stability analysis of (1.7). Therefore, one need make additional assumptions on the semigroup generated by $A$. Note that in finite dimensional spaces, the spectrum set of $A$ is always bounded. In fact, we have the following result which clearly implies the fact that for finite dimensional equations, the exponential stability cannot be destroyed by small delays.

Theorem 1.1 Assume that A generates a norm continuous $C_{0}$-semigroup $e^{t A}, t \geq 0$, i.e., $e^{\cdot A}:[0, \infty) \rightarrow \mathscr{L}(H)$ is continuous, and the semigroup generated by $A+B$ is exponentially stable in $H$. Then, there exists a constant $r_{0}>0$ such that the system (1.7) is exponentially stable for all $r \in\left(0, r_{0}\right)$.

Proof See, e.g., the proofs of Theorem 7.5 in [2].

In this work, we want to consider the sensitivity problem of pathwise stability to small delays for stochastic retarded evolution equations in Hilbert spaces. Based on the above statements, especially Theorem 1.1, let us first consider a concrete example of linear stochastic partial differential equations to motivate our theory, 


$$
\left\{\begin{array}{l}
\mathrm{d} y(t, \xi)=\frac{\partial^{2}}{\partial \xi^{2}} y(t, \xi) \mathrm{d} t+\alpha_{0} y(t, \xi) \mathrm{d} t+\sigma y(t, \xi) \mathrm{d} w(t), \quad t \geq 0, \quad \xi \in[0, \pi] \\
y(t, 0)=y(t, \pi)=0, \quad t \geq 0 \\
y(0, \cdot)=\phi_{0}(\cdot) \in H=L^{2}(0, \pi)
\end{array}\right.
$$

where $\alpha_{0}, \sigma \in \mathbb{R}$ and $w$ is a standard real Brownian motion.

It is not difficult to get, e.g., by Example 2.1, that if

$$
\alpha_{0}-1<\sigma^{2} / 2
$$

the null solution of (1.8) is exponentially stable almost surely. Further, let $r>0$ and consider a time delay version of (1.8) in the form

$$
\left\{\begin{array}{l}
\mathrm{d} y(t, \xi)=\frac{\partial^{2}}{\partial \xi^{2}} y(t, \xi)+\alpha_{0} y(t-r, \xi) \mathrm{d} t+\sigma y(t, \xi) \mathrm{d} w(t), \quad t \geq 0, \quad \xi \in[0, \pi], \\
y(t, 0)=y(t, \pi)=0, \quad t \geq 0, \\
y(0, \cdot)=\phi_{0}(\cdot) \in L^{2}(0, \pi), y_{0}(\cdot, \cdot)=\phi_{1}(\cdot, \cdot) \in L^{2}\left([-r, 0] ; L^{2}(0, \pi)\right),
\end{array}\right.
$$

where $\left(\phi_{0}, \phi_{1}\right)$ is some properly given initial datum. Here, the problem we want to address is whether, under the condition $\alpha_{0}-1<\sigma^{2} / 2$, the null solution of Eq. (1.10) secures the same pathwise exponential stability, at least for sufficiently small delay parameter $r>0$.

Note that this problem cannot be addressed by the usual method dealing with pathwise exponential stability, i.e., first considering moment stability and then establishing pathwise exponential stability. To illustrate this, let us consider a simple example of one-dimensional stochastic differential equation,

$$
\left\{\begin{array}{l}
\mathrm{d} y(t)=\beta y(t) \mathrm{d} t+\sigma y(t) \mathrm{d} w(t), \quad t \geq 0 \\
y(0)=y_{0} \in \mathbb{R}
\end{array}\right.
$$

where $\beta, \sigma \in \mathbb{R}$. It is known that

$$
\limsup _{t \rightarrow \infty} \frac{\log |y(t)|}{t}=\beta-\frac{\sigma^{2}}{2} \quad \text { a.s. }
$$

Thus, the null solution is exponentially stable if and only if $\beta<\sigma^{2} / 2$. On the other hand, we know by a simple calculation that

$$
\limsup _{t \rightarrow \infty} \frac{\log \mathbb{E}|y(t)|^{2}}{t}=2 \beta+\sigma^{2} .
$$


Thus, the null solution is mean square exponentially stable if and only if $\beta<-\sigma^{2} / 2$, a condition which is much more restrictive than $\beta<\sigma^{2} / 2$.

In this work, we shall employ a different method to address the pathwise sensitivity problem to small delays. In particular, as a by-product of the general theory established later on, we shall answer this question affirmatively for (1.10).

\section{Pathwise Exponential Stability}

Let $H, K$ be two Hilbert spaces with their norms $\|\cdot\|_{H},\|\cdot\|_{K}$ and inner products $\langle\cdot, \cdot\rangle_{H},\langle\cdot, \cdot\rangle_{K}$, respectively. We denote by $\mathscr{L}(K, H)$ the space of all bounded and linear operators from $K$ into $H$. If $H=K$, we simply denote $\mathscr{L}(H, H)$ by $\mathscr{L}(H)$. Let $\{\Omega, \mathscr{F}, \mathbb{P}\}$ be a complete probability space equipped with some filtration $\left\{\mathscr{F}_{t}\right\}_{t \geq 0}$. Let $\left\{W_{Q}(t), t \geq 0\right\}$ denote a $K$-valued $\left\{\mathscr{F}_{t}\right\}_{t \geq 0}$-Wiener process defined on $\{\Omega, \mathscr{F}, \mathbb{P}\}$ with covariance operator $Q$ satisfying that

$$
\mathbb{E}\left\langle W_{Q}(t), u\right\rangle_{K}\left\langle W_{Q}(s), v\right\rangle_{K}=(t \wedge s)\langle Q u, v\rangle_{K} \text { for all } u, v \in K
$$

where $Q$ is a positive, self-adjoint and trace class operator, $\operatorname{Tr}(Q)<\infty$, on $K$. In particular, we shall call such $W_{Q}(t), t \geq 0$, a $K$-valued $Q$-Wiener process with respect to $\left\{\mathscr{F}_{t}\right\}_{t \geq 0}$. We introduce the subspace $K_{Q}=\operatorname{Ran} Q^{1 / 2}$, the range of $Q^{1 / 2}$, of $K$ and let $\mathscr{L}_{2}=\mathscr{L}_{2}\left(K_{Q}, H\right)$ denote the space of all Hilbert-Schmidt operators from $K_{Q}$ into $H$.

Consider the following linear stochastic evolution equation

$$
\left\{\begin{array}{l}
\mathrm{d} y(t)=A y(t) \mathrm{d} t+B y(t) \mathrm{d} W(t), \quad t \geq 0 \\
y(0)=y_{0} \in H
\end{array}\right.
$$

where $A: \mathscr{D}(A) \subset H \rightarrow H$ generates a $C_{0}$-semigroup $e^{t A}, t \geq 0, B \in \mathscr{L}\left(H, \mathscr{L}_{2}\right)$, and $W(t), t \geq 0$, is a $K$-valued $Q$-Wiener process.

Lemma 2.1 Assume that A generates an exponentially stable $C_{0}$-semigroup $e^{t A}, t \geq$ 0 , such that

$$
\left\|e^{t A}\right\| \leq M e^{-\lambda t}, \quad t \geq 0
$$

for $M \geq 1, \lambda>0$ with $M^{2}\|B\|^{2}<2 \lambda$ where $\|B\|:=\|B\|_{\mathscr{L}\left(H, \mathscr{L}_{2}\right) .}$ Then, for any self-adjoint, nonnegative operator $L \in \mathscr{L}(H)$, there exists a unique nonnegative, self-adjoint operator $P \in \mathscr{L}(H)$ such that

$$
\langle A x, P x\rangle_{H}+\langle P x, A x\rangle_{H}+\langle\Delta(P) x, x\rangle_{H}=-\langle L x, x\rangle_{H} \quad \text { for any } \quad x \in \mathscr{D}(A),
$$


where $\Delta(P) \in \mathscr{L}(H)$ is the unique operator defined by the relation

$$
\langle x, \Delta(P) y\rangle_{H}:=\operatorname{Tr}\left\{P B(x) Q^{1 / 2}\left(B(y) Q^{1 / 2}\right)^{*}\right\}, \quad x, \quad y \in H, \quad P \in \mathscr{L}(H) .
$$

In this case, the operator $P$ satisfies

$$
P=\int_{0}^{\infty} e^{t A^{*}}(\Delta(P)+L) e^{t A} \mathrm{~d} t .
$$

Proof Define a series of operators $\{P(j)\}_{j \geq 0}$ in $H$ by

$$
P(0)=0, \quad P(j+1)=\int_{0}^{\infty} e^{t A^{*}}(\Delta(P(j))+L) e^{t A} \mathrm{~d} t, \quad j \in \mathbb{N}_{+} .
$$

It is immediate to see that this series is a contraction since

$$
\begin{aligned}
\|P(j+1)-P(j)\| & =\left\|\int_{0}^{\infty} e^{t A^{*}} \Delta(P(j)-P(j-1)) e^{t A} \mathrm{~d} t\right\| \\
& \leq M^{2}\|B\|^{2} \int_{0}^{\infty} e^{-2 \lambda t} \mathrm{~d} t\|P(j)-P(j-1)\| \\
& =\frac{M^{2}\|B\|^{2}}{2 \lambda}\|P(j)-P(j-1)\|
\end{aligned}
$$

and $M^{2}\|B\|^{2} / 2 \lambda<1$. Hence, there exists a unique fixed point $P \in \mathscr{L}(H)$ so that, letting $j \rightarrow \infty$ in (2.5), we obtain the relation (2.4).

To show the equality (2.2), let us consider the following backward linear operator differential equation

$$
\left\{\begin{array}{l}
\frac{d}{\mathrm{~d} t}\langle P(t) x, x\rangle_{H}+\langle A x, P(t) x\rangle_{H}+\langle P(t) x, A x\rangle_{H}+\langle[L+\Delta(P(t))] x, x\rangle_{H}=0, \\
x \in \mathscr{D}(A), \quad 0 \leq t \leq T, \\
P(T)=G,
\end{array}\right.
$$

where $G \in \mathscr{L}(H)$ is a nonnegative and self-adjoint operator in $H$ and $T \geq 0$, and the associated integral equation

$$
\begin{aligned}
P(t) x= & \int_{t}^{T} e^{(s-t) A^{*}}[L+\Delta(P(s))] e^{(s-t) A} x \mathrm{~d} s \\
& +e^{(T-t) A^{*}} G e^{(T-t) A} x, \quad \forall x \in H, \quad 0 \leq t \leq T .
\end{aligned}
$$

We shall show that Eqs.,(2.6) and (2.7) are actually equivalent. Indeed, first suppose that $P(t), t \geq 0$, satisfies (2.7). Then, by differentiating $\langle P(t) x, x\rangle_{H}, x \in \mathscr{D}(A)$, we obtain (2.6) immediately. 
Conversely, suppose $P(t), t \geq 0$, satisfies (2.6). Let $x \in \mathscr{D}(A)$ and $0<s \leq t$, and then $\left\langle P(t) e^{(t-s) A} x, e^{(t-s) A} x\right\rangle_{H}$ is differentiable in $t$ and

$$
\begin{aligned}
\frac{d}{\mathrm{~d} t}\left\langle P(t) e^{(t-s) A} x, e^{(t-s) A} x\right\rangle_{H}= & -\left\langle A e^{(t-s) A} x, P(t) e^{(t-s) A} x\right\rangle_{H}-\left\langle P(t) e^{(t-s) A} x,\right. \\
& \left.A e^{(t-s) A} x\right\rangle_{H} \\
& +\left\langle P(t) e^{(t-s) A} x, A e^{(t-s) A} x\right\rangle_{H}+\left\langle A e^{(t-s) A} x,\right. \\
& \left.P(t) e^{(t-s) A} x\right\rangle_{H} \\
& -\left\langle[L+\Delta(P(t))] e^{(t-s) A} x, e^{(t-s) A} x\right\rangle_{H} \\
= & -\left\langle[L+\Delta(P(t))] e^{(t-s) A} x, e^{(t-s) A} x\right\rangle_{H} .
\end{aligned}
$$

By integrating (2.8) in $t$ from $s$ to $T$, we then obtain for any $0 \leq s \leq T$ that

$$
\langle P(s) x, x\rangle_{H}=\int_{s}^{T}\left\langle[L+\Delta(P(u))] e^{(u-s) A} x, e^{(u-s) A} x\right\rangle_{H} \mathrm{~d} u \quad \forall x \in \mathscr{D}(A) .
$$

Since $\mathscr{D}(A)$ is dense in $H$, the relation (2.7) follows easily from (2.9). Last, since the semigroup $e^{t A}, t \geq 0$, is exponentially stable and $P$ is the unique solution of (2.4), one can see that the family of nonnegative, self-adjoint operators $P(t) \equiv P \in \mathscr{L}(H)$ is the unique solution of (2.7) with $t=0, T=+\infty$ and $G=P$. Therefore, $P$ satisfies (2.6) with $G=P$ which is exactly the equality (2.2). Thus, the proof is complete.

Lemma 2.2 Suppose that A generates a $C_{0}$-semigroup $e^{t A}, t \geq 0$, such that

$$
\left\|e^{t A}\right\| \leq M e^{-\lambda t}, \quad t \geq 0
$$

for $M>0, \lambda>0$ with $M^{2}\|B\|^{2}<2 \lambda$. Let $A_{n}$ be the Yosida approximation of $A$, i.e., $A_{n}=n R(n, A) A, R(n, A)=(n I-A)^{-1}, n \in \rho(A)$, the resolvent set of $A$, and $L \in \mathscr{L}(H)$ be a nonnegative, self-adjoint operator. For $n \in \rho(A)$, let $P_{n} \geq 0$ denote the unique solution to Lyapunov equation

$$
A_{n}^{*} P_{n}+P_{n} A_{n}+\Delta\left(P_{n}\right)=-L .
$$

Then, as $n \rightarrow \infty$,

$$
\left\langle x, P_{n} y\right\rangle_{H} \rightarrow\langle x, P y\rangle_{H} \quad \text { for any } \quad x, y \in H,
$$

where $P \in \mathscr{L}(H)$ is the unique nonnegative solution to

$$
A^{*} P+P A+\Delta(P)=-L .
$$


Proof Recall that (see (A.13) in [4])

$$
\left\|e^{t A_{n}}\right\| \leq M e^{-\frac{n \lambda t}{n-\lambda}}, \quad n>\lambda, \quad t \geq 0
$$

Thus, the exponential stability of $e^{t A}$ implies the same property of $e^{t A_{n}}$ for $n \in \rho(A)$. By virtue of Lemma 2.1, for any self-adjoint, nonnegative operator $L \in \mathscr{L}(H)$, there exists a unique solution $P_{n} \in \mathscr{L}(H)$ to (2.10) for $n \in \rho(A)$.

For each $n \in \mathbb{N}$, we construct a sequence

$$
P_{n}(0):=0 \quad \text { and } \quad P_{n}(j+1):=\int_{0}^{\infty} e^{t A_{n}^{*}}\left(\Delta\left(P_{n}(j)\right)+L\right) e^{t A_{n}} \mathrm{~d} t, \quad j \in \mathbb{N},
$$

and in a similar way, let

$$
P(0):=0 \quad \text { and } \quad P(j+1):=\int_{0}^{\infty} e^{t A^{*}}(\Delta(P(j))+L) e^{t A} \mathrm{~d} t, \quad j \in \mathbb{N} .
$$

We show that for all $x, y \in H$ and $j \in \mathbb{N}$, there is the relation $\left\langle x,\left(P_{n}(j)-\right.\right.$ $P(j)) y\rangle_{H} \rightarrow 0$ as $n \rightarrow \infty$. Indeed, we can see this by induction. For $j=0$, the claim holds trivially. Suppose now that the claim holds for $j=k-1, k \geq 1$. Let $x, y \in H$, and then for $j=k$,

$$
\begin{aligned}
& \left|\left\langle x,\left(P_{n}(k)-P(k)\right) y\right\rangle_{H}\right| \\
& =\mid \int_{0}^{\infty}\left\langle e^{t A_{n}} x,\left(\Delta\left(P_{n}(k-1)\right)+L\right) e^{t A_{n}} y\right\rangle_{H} \mathrm{~d} t \\
& -\int_{0}^{\infty}\left\langle e^{t A} x,(\Delta(P(k-1))+L) e^{t A} y\right\rangle_{H} \mathrm{~d} t \mid \\
& \leq \int_{0}^{\infty}\left|\left\langle e^{t A_{n}} x, \Delta\left(P_{n}(k-1)-P(k-1)\right) e^{t A_{n}} y\right\rangle_{H}\right| \mathrm{d} t \\
& +\mid\left\langle x, \int_{0}^{\infty} e^{t A_{n}^{*}}(\Delta(P(k-1))+L) e^{t A_{n}} y \mathrm{~d} t\right. \\
& \left.-\int_{0}^{\infty} e^{t A^{*}}(\Delta(P(k-1))+L) e^{t A} y \mathrm{~d} t\right\rangle_{H} \\
& =: I_{1}(n)+I_{2}(n) \text {. }
\end{aligned}
$$

By induction hypothesis, we have

$$
\left\langle x,\left(P_{n}(k-1)-P(k-1)\right) y\right\rangle_{H} \rightarrow 0, \quad \forall x, \quad y \in H, \quad \text { as } n \rightarrow \infty .
$$


This fact further implies that $I_{1}(n) \rightarrow 0$ as $n \rightarrow \infty$ by Dominated Convergence Theorem. For $I_{2}(n)$, we may similarly have that

$$
\begin{aligned}
\|\left\langle x, \int_{0}^{\infty}\left(e^{t A_{n}^{*}}(\Delta(P(k-1))+L) e^{t A_{n}} y-e^{t A^{*}}(\Delta(P(k-1))+L) e^{t A} y\right) \mathrm{d} t\right\rangle_{H} \mid \\
=\mid \int_{0}^{\infty}\left\langle e^{t A_{n}} x,(\Delta(P(k-1))+L)\left(e^{t A_{n}}-e^{t A}\right) y\right\rangle_{H} \\
\quad+\left\langle\left(e^{t A_{n}}-e^{t A}\right) x,(\Delta(P(k-1))+L) e^{t A} y\right\rangle_{H} \mathrm{~d} t \mid \\
\leq \int_{0}^{\infty}\left\|e^{t A_{n}}\right\| \cdot\|x\|_{H}\|\Delta(P(k-1))+L\| \cdot\left\|\left(e^{t A_{n}}-e^{t A}\right) y\right\|_{H} \mathrm{~d} t \\
\quad+\int_{0}^{\infty}\left\|\left(e^{t A_{n}}-e^{t A}\right) x\right\|_{H}\|\Delta(P(k-1))+L\| \cdot\left\|e^{t A}\right\| \cdot\|y\|_{H} \mathrm{~d} t \\
\rightarrow 0 \quad \text { as } n \rightarrow \infty,
\end{aligned}
$$

by virtue of Dominated Convergence Theorem. Hence, the claim is proved by induction.

Last, by the proofs of Lemma 2.1 we have that $P(j) \rightarrow P$ and $P_{n}(j) \rightarrow P_{n}$, uniformly in $n$, as $j \rightarrow \infty$ in the norm topology of $\mathscr{L}(H)$. Therefore, we obtain that

$$
\left\langle x,\left(P_{n}-P\right) y\right\rangle_{H} \rightarrow 0 \quad \text { as } \quad n \rightarrow \infty \quad \text { for any } \quad x, y \in H
$$

by using the relation

$$
\begin{aligned}
\left|\left\langle x,\left(P_{n}-P\right) y\right\rangle_{H}\right| \leq & \left|\left\langle x,\left(P_{n}-P_{n}(j)\right) y\right\rangle_{H}\right|+\left|\left\langle x,\left(P_{n}(j)-P(j)\right) y\right\rangle_{H}\right| \\
& +\left|\langle x,(P(j)-P) y\rangle_{H}\right| .
\end{aligned}
$$

The proof is thus complete.

Now consider the following linear stochastic evolution equation on the Hilbert space $H$,

$$
\left\{\begin{array}{l}
\mathrm{d} y(t)=A y(t) \mathrm{d} t+\sum_{i=1}^{n} B_{i} y(t) \mathrm{d} w_{i}(t), \quad t \geq 0, \\
y(0)=y_{0} \in H
\end{array}\right.
$$

where $B_{i} \in \mathscr{L}(H)$ and $w_{i}(t), t \geq 0, i=1, \ldots, n$, are a group of independent, standard real-valued Brownian motions. To proceed further, we first recall two useful lemmas which are important in their own right. The first lemma is a strong law of large numbers for continuous local martingales (see, e.g., [9] or Theorem 1.3.4 in [12]), and the proof of the second is referred to that of Proposition 2.1.4 in [10]. 
Lemma 2.3 Let $M(t), t \geq 0$, be a real-valued, continuous local martingale with $M(0)=0$. If its quadratic variation $[M]$ satisfies that

$$
\limsup _{t \rightarrow \infty} \frac{[M](t)}{t}<\infty \quad \text { a.s. }
$$

then there is

$$
\lim _{t \rightarrow \infty} \frac{M(t)}{t}=0 \quad \text { a.s. }
$$

Lemma 2.4 Suppose that $A$ generates a $C_{0}$-semigroup $e^{t A}, t \geq 0$, on the Hilbert space $H$ and $A^{*}$ is its adjoint operator. There exists a real number $\alpha \in \mathbb{R}$ such that

$$
\operatorname{Re}\langle x, A x\rangle_{H} \leq \alpha\|x\|_{H}^{2} \quad \text { for all } \quad x \in \mathscr{D}(A),
$$

and

$$
\operatorname{Re}\left\langle x, A^{*} x\right\rangle_{H} \leq \alpha\|x\|_{H}^{2} \quad \text { for all } \quad x \in \mathscr{D}\left(A^{*}\right),
$$

if and only if A generates a pseudo-contraction $C_{0}$-semigroup $e^{t A}, t \geq 0$, in the sense that

$$
\left\|e^{t A}\right\| \leq e^{\alpha t} \quad \text { for all } \quad t \geq 0
$$

Proposition 2.1 Let $B=\left(B_{1}, \ldots, B_{n}\right)$. Suppose that there exist $\beta=\left(\beta_{1}, \ldots, \beta_{n}\right)$ $\in \mathbb{R}^{n}, \lambda>0$ and $M \geq 1$ such that the $C_{0}$-semigroup $e^{t(A+\beta \cdot B)}$ generated by $A+\beta \cdot B$ satisfies

$$
\left\|e^{t(A+\beta \cdot B)}\right\| \leq M e^{-\lambda t}, \quad t \geq 0,
$$

and

$$
\frac{1}{4} \sum_{i=1}^{n} \beta_{i}^{2}+\frac{M^{2}}{2} \sum_{i=1}^{n}\left\|B_{i}\right\|^{2}<\lambda
$$

then the mild solution of (2.13) is almost sure exponentially stable with

$$
\limsup _{t \rightarrow \infty} \frac{1}{t} \log \|y(t)\|_{H} \leq-\left(\lambda-\frac{1}{4} \sum_{i=1}^{n} \beta_{i}^{2}-\frac{M^{2}}{2} \sum_{i=1}^{n}\left\|B_{i}\right\|^{2}\right) \quad \text { a.s. }
$$

Proof Choose arbitrarily

$$
\gamma \in\left(0, \lambda-\frac{1}{4} \sum_{i=1}^{n} \beta_{i}^{2}-\frac{M^{2}}{2} \sum_{i=1}^{n}\left\|B_{i}\right\|^{2}\right)
$$


Let us put $\|\beta\|^{2}:=\sum_{i=1}^{n} \beta_{i}^{2}$ and $\Lambda=A+\beta \cdot B+\frac{\|\beta\|^{2}}{4}+\gamma$, and then $\Lambda$ is the generator of a $C_{0}$-semigroup $T(t)$ which satisfies

$$
\|T(t)\| \leq M e^{-\left(\lambda-\|\beta\|^{2} / 4-\gamma\right) t}, \quad t \geq 0 .
$$

For each $m \in \mathbb{N}$, let $\Lambda_{m}:=A_{m}+\beta \cdot B+\frac{\|\beta\|^{2}}{4}+\gamma$ where $A_{m}$ is the Yosida approximation of $A$. Without loss of generality, it is assumed that for all $m \geq 1$, we have

$$
\left\|T_{m}(t)\right\| \leq M e^{-\left(\lambda-\|\beta\|^{2} / 4-\gamma\right) t}, \quad t \geq 0
$$

Since semigroups $T(\cdot)$ and $T_{m}(\cdot), m \geq 1$, are exponentially stable, by virtue of (2.17) and Lemma 2.1, one can find for $m \in \mathbb{N}$ and $\Delta(I) \in \mathscr{L}^{+}(H)$ a unique solution $Q_{m} \in \mathscr{L}(H)$ to the following Lyapunov equations

$$
\Lambda_{m}^{*} Q_{m}+Q_{m} \Lambda_{m}+\Delta\left(Q_{m}\right)=-\Delta(I)
$$

In other words, for each $m \geq 1, P_{m}=Q_{m}+I \in \mathscr{L}(H)$ is the unique solution to the Lyapunov equation

$$
\Lambda_{m}^{*}\left(P_{m}-I\right)+\left(P_{m}-I\right) \Lambda_{m}+\Delta\left(P_{m}-I\right)=-\Delta(I)
$$

In an analogous manner, let $P \in \mathscr{L}(H)$ be the unique solution to

$$
\Lambda^{*}(P-I)+(P-I) \Lambda+\Delta(P-I)=-\Delta(I) .
$$

Then, by virtue of Lemmas 2.1 and 2.2, it is easy to show

$$
P_{m} \geq I, \quad m \in \mathbb{N}, \quad P \geq I \quad \text { and } \quad\left\langle P_{m} x, y\right\rangle_{H} \rightarrow\langle P x, y\rangle_{H}, \quad \text { as } m \rightarrow \infty,
$$

for all $x, y \in H$. By the definition of $\Lambda_{m}, m \geq 1$, and Lemma 2.4, it follows that

$$
\left\langle\left(\Lambda_{m}+\Lambda_{m}^{*}\right) x, x\right\rangle_{H} \leq 0 \quad \text { for any } \quad x \in H .
$$

Hence, by a direct computation we find from relation (2.19) that

$$
\begin{aligned}
& \left(A_{m}+\beta \cdot B\right)^{*} P_{m}+P_{m}\left(A_{m}+\beta \cdot B\right)+\Delta\left(P_{m}\right)+\frac{\|\beta\|^{2}}{2} P_{m}+2 \gamma P_{m} \leq 0 \\
& \text { for each } \quad m \in \mathbb{N} .
\end{aligned}
$$


In other words, for any $m \in \mathbb{N}$ and $x \in H$, we have

$$
\begin{aligned}
\left\langle P_{m} A_{m} x, x\right\rangle_{H}+ & \left\langle A_{m} x, P_{m} x\right\rangle_{H}+\left\langle\Delta\left(P_{m}\right) x, x\right\rangle_{H}+2 \gamma\left\langle P_{m} x, x\right\rangle_{H} \\
& \leq-\sum_{i=1}^{n} \beta_{i}\left\langle P_{m} x, B_{i} x\right\rangle_{H}-\sum_{i=1}^{n} \beta_{i}\left\langle B_{i} x, P_{m} x\right\rangle_{H} \\
& -\frac{\|\beta\|^{2}}{2}\left\langle P_{m} x, x\right\rangle_{H},
\end{aligned}
$$

which further implies that for any $m \in \mathbb{N}$ and $x \in H$,

$$
\begin{aligned}
& \left\langle P_{m} x, x\right\rangle_{H}\left[\left\langle P_{m} x, A_{m} x\right\rangle_{H}+\left\langle A_{m} x, P_{m} x\right\rangle_{H}+\left\langle\Delta\left(P_{m}\right) x, x\right\rangle_{H}+2 \gamma\left\langle P_{m} x, x\right\rangle_{H}\right] \\
& \leq-\sum_{i=1}^{n} \beta_{i}\left\langle P_{m} x, x\right\rangle_{H}\left\langle P_{m} x, B_{i} x\right\rangle_{H}-\sum_{i=1}^{n} \beta_{i}\left\langle P_{m} x, x\right\rangle_{H}\left\langle B_{i} x, P_{m} x\right\rangle_{H} \\
& -\frac{\|\beta\|^{2}}{2}\left\langle P_{m} x, x\right\rangle_{H}^{2} \\
& \leq 2 \sum_{i=1}^{n}\left\langle P_{m} x, B_{i} x\right\rangle_{H}^{2} .
\end{aligned}
$$

On the other hand, let $y_{m}$ denote the mild solution to the equation

$$
\left\{\begin{array}{l}
\mathrm{d} y_{m}(t)=A_{m} y_{m}(t) \mathrm{d} t+\sum_{i=1}^{n} B_{i} y_{m}(t) \mathrm{d} w_{i}(t), \quad t \geq 0, \\
y_{m}(0)=y_{0},
\end{array}\right.
$$

where $m \in \mathbb{N}$. Then, there exists a sequence, still denote it by $y_{m} \in H$ (see [4]), such that

$$
\sup _{t \in[0, T]}\left\|y_{m}(t)-y(t)\right\|_{H} \rightarrow 0 \quad \text { as } m \rightarrow \infty \quad \text { a.s. }
$$

If $y_{0}=0$, then $\mathbb{P}(y(t, 0)=0)=1$ for $t \geq 0$, and the desired estimate holds trivially. Now suppose that $y_{0} \neq 0$, then by uniqueness of the solutions and strict positivity of $P_{m}, \mathbb{P}\left(\left\langle P_{m} y_{m}(t), y_{m}(t)\right\rangle_{H}=0\right)=0$ for all $t \geq 0$ (or, the reader is referred to a proof similar to that of Lemma 2.1 in [11]). Letting $\theta_{m}(t)=\left\langle P_{m} y_{m}(t), y_{m}(t)\right\rangle_{H}, t \geq 0$, and applying Itô's formula to $\log \theta_{m}(t)$, we obtain by (2.22) that for $t \geq 0$,

$$
\begin{aligned}
\log \left\langle P_{m} y_{m}(t), y_{m}(t)\right\rangle_{H} \\
=\log \left\langle P_{m} y_{0}, y_{0}\right\rangle_{H}+\int_{0}^{t}\left\{\frac { 1 } { \theta _ { m } ( s ) } \left[\left\langle P_{m} y_{m}(s), A_{m} y_{m}(s)\right\rangle_{H}\right.\right. \\
\left.\quad+\left\langle A_{m} y_{m}(s), P_{m} y_{m}(s)\right\rangle_{H}+\left\langle\Delta\left(P_{m}\right) y_{m}(s), y_{m}(s)\right\rangle_{H}\right]
\end{aligned}
$$




$$
\begin{aligned}
& \left.-\frac{2}{\theta_{m}^{2}(s)} \sum_{i=1}^{n}\left\langle P_{m} y_{m}(s), B_{i} y_{m}(s)\right\rangle_{H}^{2}\right\} d s \\
& +\int_{0}^{t} \frac{2}{\theta_{m}(s)} \sum_{i=1}^{n}\left\langle P_{m} y_{m}(s), B_{i} y_{m}(s) \mathrm{d} w_{i}(s)\right\rangle_{H} \\
& \leq \log \left\langle P_{m} y_{0}, y_{0}\right\rangle_{H}-2 \gamma t+2 \int_{0}^{t} \sum_{i=1}^{n}\left\langle\frac{P_{m} y_{m}(s)}{\left\langle P_{m} y_{m}(s), y_{m}(s)\right\rangle_{H}}, B_{i} y_{m}(s) \mathrm{d} w_{i}(s)\right\rangle_{H}
\end{aligned}
$$

Letting $m \rightarrow \infty$ and using (2.20) and (2.24), we have from (2.25) that

$$
\begin{aligned}
& \log \langle P y(t), y(t)\rangle_{H} \leq \log \left\langle P y_{0}, y_{0}\right\rangle_{H}-2 \gamma t \\
& +2 \sum_{i=1}^{n} \int_{0}^{t}\left\langle\frac{P y(s)}{\langle P y(s), y(s)\rangle_{H}}, B y(s) \mathrm{d} w_{i}(s)\right\rangle_{H} .
\end{aligned}
$$

On the other hand, it is easy to see that the stochastic integral

$$
\int_{0}^{t}\left\langle\frac{2 P y(s)}{\langle P y(s), y(s)\rangle_{H}}, B_{i} y(s) \mathrm{d} w_{i}(s)\right\rangle_{H}, \quad t \geq 0, \quad i=1, \ldots, n,
$$

is a real continuous local martingale, and since $\langle P x, x\rangle_{H} \geq\|x\|_{H}^{2}$ for any $x \in H$, it follows that

$$
\sup _{x \neq 0, x \in H} \frac{\left\langle P x, B_{i} x\right\rangle_{H}^{2}}{\langle P x, x\rangle_{H}^{2}} \leq \sup _{x \neq 0, x \in H} \frac{\|P\|^{2}\left\|B_{i}\right\|^{2}\|x\|_{H}^{4}}{\|x\|_{H}^{4}}<\infty .
$$

Thus, one can employ Lemma 2.3 to get that for each $i \in\{1, \ldots, n\}$,

$$
\frac{1}{t} \int_{0}^{t}\left\langle\frac{2 P y(s)}{\langle P y(s), y(s)\rangle_{H}}, B_{i} y(s) \mathrm{d} w_{i}(s)\right\rangle_{H} \rightarrow 0 \quad \text { as } \quad t \rightarrow \infty \quad \text { a.s. }
$$

which, in addition to (2.26), immediately implies that

$$
\limsup _{t \rightarrow \infty} \frac{1}{t} \log \|y(t)\|_{H}^{2} \leq \limsup _{t \rightarrow \infty} \frac{1}{t} \log \langle P y(t), y(t)\rangle_{H} \leq-2 \gamma \quad \text { a.s. }
$$

Last, letting $\gamma \rightarrow \lambda-\frac{1}{4} \sum_{i=1}^{n} \beta_{i}^{2}-\frac{M^{2}}{2} \sum_{i=1}^{n}\left\|B_{i}\right\|^{2}$, we obtain the desired result.

Example 2.1 Consider the following linear stochastic differential equation in $H$,

$$
\left\{\begin{array}{l}
\mathrm{d} y(t)=A y(t) \mathrm{d} t+\sigma y(t) \mathrm{d} w(t), \quad t \geq 0 \\
y(0)=y_{0} \in H
\end{array}\right.
$$


where $w(\cdot)$ is a standard real Brownian motion and $A$ generates a $C_{0}$-semigroup $e^{t A}$, $t \geq 0$, satisfying $\left\|e^{t A}\right\| \leq e^{\alpha t}$ for some $\alpha \in \mathbb{R}$ and $\sigma \in \mathbb{R}$. We claim that if $\alpha<\sigma^{2} / 2$, the mild solution $y$ to (2.27) is exponentially stable almost surely.

To see this, it suffices by Proposition 2.1 to find $\lambda>0$ and $\beta \in \mathbb{R}$ such that

$$
-\lambda>\alpha+\beta \sigma \quad \text { and } \quad \sigma^{2}+\frac{\beta^{2}}{2}-2 \lambda<0 .
$$

However, this is immediate since we can take $\beta=-2 \sigma$ and $\lambda=3 \sigma^{2} / 2+\varepsilon$ with $\varepsilon>0$ small enough by using the condition $\alpha<\sigma^{2} / 2$ and the mild solution $y$ is pathwise exponentially stable with

$$
\limsup _{t \rightarrow \infty} \frac{1}{t} \log \|y(t)\|_{H} \leq \alpha-\frac{\sigma^{2}}{2} \quad \text { a.s. }
$$

\section{Stochastic Delay Evolution Equations}

Let $H$ be a real Hilbert space and $V$ be another Hilbert space such that $V$ is dense in $H$ and the inclusion map $V \hookrightarrow H$ is continuous. The norms and inner products of $H, V$ are denoted by $\|\cdot\|_{H},\|\cdot\|_{V}$ and $\langle\cdot, \cdot\rangle_{H},\langle\cdot, \cdot\rangle_{V}$, respectively. By identifying the dual of $H$ with $H$, we may assume that

$$
V \hookrightarrow H \hookrightarrow V^{*} \quad \text { and } \quad\|x\|_{H}^{2} \leq v\|x\|_{V}^{2}, \quad x \in V
$$

for some constant $v \geq 1$. Moreover, we have for any $T \geq 0$ that

$$
L^{2}([0, T] ; V) \cap W^{1,2}\left([0, T] ; V^{*}\right) \subset C([0, T] ; H)
$$

where $W^{1,2}\left([0, T] ; V^{*}\right)$ is the classic Sobolev space consisting of all functions $y \in L^{2}\left([0, T] ; V^{*}\right)$ such that $y$ and its first-order distributional derivatives are in $L^{2}\left([0, T] ; V^{*}\right)$ and $C([0, T] ; H)$ is the space of all continuous functions from $[0, T]$ into $H$, respectively. The duality pair between $V$ and $V^{*}$ is denoted by $\langle\langle\cdot, \cdot\rangle\rangle_{V, V^{*}}$. Let $a: V \times V \rightarrow \mathbb{R}$ be a bounded bilinear form satisfying Gårding's inequality

$$
a(x, x) \leq-\alpha\|x\|_{V}^{2}+\lambda\|x\|_{H}^{2}, \quad \forall x \in V,
$$

for some $\alpha>0$ and $\lambda \in \mathbb{R}$. Let $A$ be the operator associated with this form by

$$
a(x, y)=\langle\langle x, A y\rangle\rangle_{V, V^{*}}, \quad x, y \in V .
$$

Then, $A \in \mathscr{L}\left(V, V^{*}\right)$, the family of all bounded and linear operators from $V$ to $V^{*}$. The realization of $A$ in $H$, which is the restriction of $A$ to the domain $\mathscr{D}(A)=\{x \in$ $V: A x \in H\}$, is also denoted by $A$. Hence, $\langle x, A y\rangle_{H}=\langle\langle x, A y\rangle\rangle_{V, V^{*}}$ for all $x \in V$ and $y \in V$ with $A y \in H$. It is well known that $A$ generates an analytic $C_{0}$-semigroup $e^{t A}, t \geq 0$, in $V^{*}$ such that $e^{t A}: V^{*} \rightarrow V$ for each $t>0$. In this work, we always 
suppose, for simplicity, that $\lambda=0$ in (3.2) and $A$ generates an exponentially stable $C_{0}$-semigroup with $\left\|e^{t A}\right\| \leq e^{-\alpha t}$ for all $t \geq 0$.

Let $r>0, T \geq 0$ and $\overline{\mathcal{H}}=H \times L^{2}([-r, 0] ; V)$. We first consider the following deterministic retarded differential equation in space $V^{*}$,

$$
\left\{\begin{array}{l}
\mathrm{d} y(t)=A y(t) \mathrm{d} t+A_{1} y(t-r) \mathrm{d} t+\int_{-r}^{0} A_{2}(\theta) y(t+\theta) \mathrm{d} \theta \mathrm{d} t, \quad t \in[0, T], \\
y(0)=\phi_{0}, \quad y(\theta)=\phi_{1}(\theta), \quad \theta \in[-r, 0], \quad\left(\phi_{0}, \phi_{1}\right) \in \mathcal{H},
\end{array}\right.
$$

where $A_{1} \in \mathscr{L}\left(V, V^{*}\right)$ and $A_{2}(\cdot) \in L^{2}\left([-r, 0] ; \mathscr{L}\left(V, V^{*}\right)\right)$. In particular, a function $y \in L^{2}([-r, T] ; V) \cap W^{1,2}\left([0, T] ; V^{*}\right)$ which satisfies (3.3) is called a strong solution of (3.3) in [ $-r, T]$. It has been shown (see [7] or [8]) that there exists a unique solution $y$ of (3.3) such that

$$
y \in L^{2}([0, T] ; V) \cap W^{1,2}\left([0, T] ; V^{*}\right) \subset C([0, T] ; H) .
$$

In association with this solution $y$, we may define a family of operators $\mathcal{T}(t), t \geq 0$, on $\mathcal{H}$ by

$$
\mathcal{T}(t) \phi=\left(y(t ; \phi), y_{t}(\phi)\right), \quad t \geq 0, \quad \phi \in \mathcal{H}
$$

Then, it has been shown (see [7]) that $\mathcal{T}(t), t \geq 0$, is actually a $C_{0}$-semigroup on $\mathcal{H}$. Let $\mathcal{A}$ be the infinitesimal generator of $\mathcal{T}(t)$ or $e^{t \mathcal{A}}, t \geq 0$, and then $\mathcal{A}$ is completely described by the following theorem.

Theorem 3.1 The operator $\mathcal{A}$ is given by

$$
\begin{aligned}
\mathscr{D}(\mathcal{A})=\left\{\phi=\left(\phi_{0}, \phi_{1}\right):\right. & \phi_{1} \in W^{1,2}([-r, 0] ; V), \phi_{0}=\phi_{1}(0), \\
& \left.A \phi_{0}+A_{1} \phi_{1}(-r)+\int_{-r}^{0} A_{2}(\theta) \phi_{1}(\theta) d \theta \in H\right\},
\end{aligned}
$$

and for any $\phi=\left(\phi_{0}, \phi_{1}\right) \in \mathscr{D}(\mathcal{A})$,

$$
\mathcal{A} \phi=\left(A \phi_{0}+A_{1} \phi_{1}(-r)+\int_{-r}^{0} A_{2}(\theta) \phi_{1}(\theta) d \theta, \frac{d \phi_{1}(\theta)}{d \theta}\right) .
$$

Note that $C_{0}$-semigroup $e^{t \mathcal{A}}, t \geq 0$, allows us to transfer the exponential stability problem of the time delay system (3.3) to that one of a non-time delay system. To see this, we can rewrite (3.3) as a Cauchy problem,

$$
\left\{\begin{array}{l}
\mathrm{d} Y(t)=\mathcal{A} Y(t) \mathrm{d} t, \quad t \geq 0 \\
Y(0)=\phi \in \mathcal{H}
\end{array}\right.
$$


where $Y(t)=(y(t), y(t+\cdot))$ is the lift-up function of $y(t), t \geq 0$. To show the exponential stability of the null solution of (3.3) in $H$, it suffices to study the corresponding exponential stability of system (3.7). Indeed, assume that the null solution of (3.7) is exponentially stable, and then there exist constants $M>0$ and $\mu>0$ such that

$$
\begin{aligned}
\|y(t, \phi)\|_{H}^{2} & \leq\|y(t, \phi)\|_{H}^{2}+\int_{-r}^{0}\|y(t+\theta, \phi)\|_{V}^{2} \mathrm{~d} \theta \\
& =\|Y(t, \phi)\|_{\mathcal{H}}^{2} \\
& \leq M e^{-\mu t}\|\phi\|_{\mathcal{H}}^{2}, \quad t \geq 0 .
\end{aligned}
$$

Hence, it is natural to consider exponential stability of the non-time delay system (3.7) rather than time delay one (3.3). To establish the exponential stability of the $C_{0}$-semigroup $e^{t \mathcal{A}}, t \geq 0$, one may want to develop a dissipative operator program by using Lemma 2.4 . However, the following example shows some difficulties in association with this scheme.

Let $\alpha>0, \beta \in \mathbb{R}$ and consider a real-valued differential equation

$$
\left\{\begin{array}{l}
\mathrm{d} u(t)=-\alpha u(t) \mathrm{d} t+\beta u(t-1) \mathrm{d} t, \quad t \geq 0, \\
u(\theta)=1, \theta \in[-1,0] .
\end{array}\right.
$$

If $\beta=0$, the solution $u(t)=e^{-\alpha t}, t \geq 0$, which is exponentially stable. Otherwise, it is clear that $\mathcal{H}=\mathbb{R} \times L^{2}([-1,0] ; \mathbb{R})$ equipped with the usual inner product. If we take the above viewpoint to consider system (3.7), then it turns out in this case that for any number $a>0$ and $\phi \in \mathscr{D}(\mathcal{A})$,

$$
\begin{aligned}
\langle\mathcal{A} \phi, \phi\rangle_{\mathcal{H}}+a\|\phi\|_{\mathcal{H}}^{2}= & (a-\alpha) \phi_{0}^{2}+\beta \phi_{0} \phi_{1}(-1)+\frac{1}{2} \phi_{1}^{2}(0) \\
& -\frac{1}{2} \phi_{1}^{2}(-r)+a \int_{-r}^{0} \phi_{1}^{2}(\theta) \mathrm{d} \theta
\end{aligned}
$$

It is obvious that one can always find a nonzero element $\phi \in \mathscr{D}(\mathcal{A})$ with $\phi_{0}=\phi_{1}(0)=$ $\phi_{1}(-r)=0$ such that

$$
\langle\mathcal{A} \phi, \phi\rangle_{\mathcal{H}}+a\|\phi\|_{\mathcal{H}}^{2}>0
$$

In other words, it is impossible, according to Lemma 2.4, to find a constant $a>0$ such that $\left\|e^{t \mathcal{A}}\right\| \leq e^{-a t}, t \geq 0$ in spite of the fact that $\left\|e^{t A}\right\| \leq e^{-\alpha t}, t \geq 0$. To avoid this difficulty, we can introduce an equivalent inner product $(\cdot, \cdot)_{\mathcal{H}}$ or norm $|\cdot|$ to the canonical one $\langle\cdot, \cdot\rangle_{\mathcal{H}}$ or $\|\cdot\|$ on $\mathcal{H}$ so as that the semigroup $e^{t \mathcal{A}}, t \geq 0$, becomes exponentially stable under the norm $\|\cdot\|$.

Theorem 3.2 Suppose that $\langle\langle x, A x\rangle\rangle_{V, V^{*}} \leq-\alpha\|x\|_{V}^{2}$ for all $x \in V$ and some $\alpha>0$. If, further, 


$$
\lambda<\alpha-\left((|\eta|(0)-|\eta|(-r)) \int_{-r}^{0} e^{-2 \lambda \theta} d|\eta|(\theta)\right)^{1 / 2} \quad \text { for some } \quad 0<\lambda<\alpha,
$$

where $|\eta|(\theta)$ is the total variation on $[-r, \theta], \theta \in[-r, 0]$, of the bounded variation function $\eta$ given by

$$
\eta(\tau)=-\mathbf{1}_{(-\infty,-r]}(\tau) A_{1}-\int_{\tau}^{0} A_{2}(\theta) d \theta, \quad \tau \in[-r, 0],
$$

then there exists a constant $M>0$ such that

$$
\left\|e^{t \mathcal{A}}\right\| \leq M e^{-\frac{\lambda}{v} t} \quad \text { for all } \quad t \geq 0
$$

where $v$ is the constant given in (3.1).

Proof We show that there exists an equivalent inner product $(\cdot, \cdot) \mathcal{H}$ on $\mathcal{H}=H \times$ $L^{2}([-r, 0] ; V)$ to the canonical one $\langle\cdot, \cdot\rangle_{\mathcal{H}}$ on $\mathcal{H}$ such that

$$
(\mathcal{A} \phi, \phi)_{\mathcal{H}} \leq-\lambda \nu^{-1}\|\phi\|_{\mathcal{H}} \quad \text { for all } \quad \phi \in \mathscr{D}(\mathcal{A})
$$

Here $(\cdot, \cdot)_{\mathcal{H}}$ is defined by

$$
(\phi, \psi)_{\mathcal{H}}:=\left\langle\phi_{0}, \psi_{0}\right\rangle_{H}+\int_{-r}^{0} \gamma(\theta)\left\langle\phi_{1}(\theta), \psi_{1}(\theta)\right\rangle_{V} \mathrm{~d} \theta, \quad \phi, \psi \in \mathcal{H},
$$

where $\gamma:[-r, 0] \rightarrow \mathbb{R}_{+}$is given by

$$
\gamma(\theta)=e^{2 \lambda \theta}\left[\alpha-\lambda-\frac{|\eta|(0)-|\eta|(-r)}{\alpha-\lambda} \int_{\theta}^{0} e^{-2 \lambda \tau} d|\eta|(\tau)\right], \quad \theta \in[-r, 0] .
$$

First, note that under the conditions of Theorem 3.2, bilinear form (3.10) does define an inner product. Indeed, both (3.9) and (3.11) imply the lower boundedness of $\gamma(\cdot)$,

$$
\begin{aligned}
& \gamma(\theta) \geq e^{-2 \lambda r}\left[\alpha-\lambda-\frac{|\eta|(0)-|\eta(-r)|}{\alpha-\lambda} \int_{-r}^{0} e^{-2 \lambda \tau} d|\eta|(\tau)\right] \\
& \quad \text { for any } \quad \theta \in[-r, 0] .
\end{aligned}
$$

This implies that $(\cdot, \cdot)_{\mathcal{H}}$ defines an inner product on $\mathcal{H}$. On the other hand, it is easy to see that for any $\phi \in \mathcal{H}$,

$$
\begin{aligned}
(\phi, \phi)_{\mathcal{H}} & =\left\|\phi_{0}\right\|_{H}^{2}+\int_{-r}^{0} \gamma(\theta)\left\|\phi_{1}(\theta)\right\|_{V}^{2} \mathrm{~d} \theta \\
& \leq\left[1+e^{2 \lambda r}(\alpha-\lambda)\right]\left(\left\|\phi_{0}\right\|_{H}^{2}+\int_{-r}^{0}\left\|\phi_{1}(\theta)\right\|_{V}^{2} \mathrm{~d} \theta\right)
\end{aligned}
$$


which implies that the inner product $(\cdot, \cdot)_{\mathcal{H}}$ is also equivalent to the canonical inner product $\langle\cdot, \cdot\rangle_{\mathcal{H}}$ on $\mathcal{H}$. On the other hand,

$$
\begin{aligned}
\left(\phi,\left(\mathcal{A}+\lambda v^{-1}\right) \phi\right)_{\mathcal{H}}= & \left\langle\phi_{0}, A \phi_{0}+\lambda v^{-1} \phi_{0}+A_{1} \phi_{1}(-r)+\int_{-r}^{0} A_{2}(\theta) \phi_{1}(\theta) \mathrm{d} \theta\right\rangle_{H} \\
& +\int_{-r}^{0} \gamma(\theta)\left\langle\phi_{1}(\theta), \dot{\phi}_{1}(\theta)+\lambda v^{-1} \phi_{1}(\theta)\right\rangle_{V} \mathrm{~d} \theta .
\end{aligned}
$$

Since $\langle x, y\rangle_{H}=\langle\langle x, y\rangle\rangle_{V, V^{*}}$ for any $x \in V, y \in H$, and

$$
\langle\langle x, A x\rangle\rangle_{V, V^{*}} \leq-\alpha\|x\|_{V}^{2} \quad \text { for any } \quad x \in V,
$$

it follows for any $\phi \in \mathscr{D}(\mathcal{A})$ that

$$
\begin{aligned}
(\phi, & \left.\left(\mathcal{A}+\lambda v^{-1}\right) \phi\right)_{\mathcal{H}} \\
\leq & \left\langle\left\langle\phi_{0}, A \phi_{0}\right\rangle\right\rangle_{V, V^{*}}+\left\langle\left\langle\phi_{0}, A_{1} \phi_{1}(-r)+\int_{-r}^{0} A_{2}(\theta) \phi_{1}(\theta) \mathrm{d} \theta\right\rangle\right\rangle_{V, V^{*}} \\
& +\int_{-r}^{0} \gamma(\theta)\left\langle\dot{\phi}_{1}(\theta), \phi_{1}(\theta)\right\rangle_{V} \mathrm{~d} \theta+\lambda\left\|\phi_{0}\right\|_{V}^{2}+\lambda \int_{-r}^{0} \gamma(\theta)\left\|\phi_{1}(\theta)\right\|_{V}^{2} \mathrm{~d} \theta \\
\leq & (\lambda-\alpha)\left\|\phi_{0}\right\|_{V}^{2}+\left\|\phi_{0}\right\|_{V} \int_{-r}^{0}\left\|\phi_{1}(\theta)\right\|_{V} d|\eta|(\theta) \\
& +\int_{-r}^{0} \gamma(\theta)\left(\frac{1}{2} \frac{d}{\mathrm{~d} \theta}\left\|\phi_{1}(\theta)\right\|_{V}^{2}+\lambda\left\|\phi_{1}(\theta)\right\|_{V}^{2}\right) \mathrm{d} \theta .
\end{aligned}
$$

By using integration by parts, one can further derive from (3.12) that for $\phi \in \mathscr{D}(\mathcal{A})$,

$$
\begin{aligned}
\left(\phi,\left(\mathcal{A}+\lambda v^{-1}\right) \phi\right)_{\mathcal{H}} \leq & (\lambda-\alpha)\left\|\phi_{0}\right\|_{V}^{2}+\left\|\phi_{0}\right\|_{V} \int_{-r}^{0}\left\|\phi_{1}(\theta)\right\|_{V} d|\eta|(\theta) \\
& +\frac{1}{2}(\alpha-\lambda)\left\|\phi_{0}\right\|_{V}^{2} \\
& -\frac{1}{2} \gamma(-r)\left\|\phi_{1}(-r)\right\|_{V}^{2}-\frac{|\eta|(0)-|\eta|(-r)}{2(\alpha-\lambda)} \int_{-r}^{0}\left\|\phi_{1}(\theta)\right\|_{V}^{2} d|\eta|(\theta) \\
\leq & -\frac{1}{2}(\alpha-\lambda)\left\|\phi_{0}\right\|_{V}^{2}+\left\|\phi_{0}\right\|_{V} \int_{-r}^{0}\left\|\phi_{1}(\theta)\right\|_{V} d|\eta|(\theta) \\
& -\frac{|\eta|(0)-|\eta|(-r)}{2(\alpha-\lambda)} \int_{-r}^{0}\left\|\phi_{1}(\theta)\right\|_{V}^{2} d|\eta|(\theta) .
\end{aligned}
$$

If $\left\|\phi_{0}\right\|_{V}=0$ or $|\eta|(0)=0$, i.e., $\eta$ is constant, it is immediate from (3.13) that

$$
\left(\phi,\left(\mathcal{A}+\lambda v^{-1}\right) \phi\right)_{\mathcal{H}} \leq 0 \quad \text { for all } \quad \phi \in \mathscr{D}(\mathcal{A})
$$


If $\left\|\phi_{0}\right\|_{V} \neq 0$ and $|\eta|(0)>0$ for $\phi \in \mathscr{D}(\mathcal{A})$, we have from (3.13) that

$$
\begin{aligned}
\left(\phi,\left(\mathcal{A}+\lambda \nu^{-1}\right) \phi\right)_{\mathcal{H}} \leq & \left\|\phi_{0}\right\|_{V}^{2} \int_{-r}^{0}\left[-\frac{\alpha-\lambda}{2(|\eta|(0)-|\eta|(-r))}+\frac{\left\|\phi_{1}(\theta)\right\|_{V}}{\left\|\phi_{0}\right\|_{V}}\right. \\
& \left.-\frac{|\eta|(0)-|\eta|(-r)}{2(\alpha-\lambda)} \frac{\left\|\phi_{1}(\theta)\right\|_{V}^{2}}{\left\|\phi_{0}\right\|_{V}^{2}}\right] d|\eta|(\theta) \\
= & \frac{\left\|\phi_{0}\right\|_{V}^{2}}{2} \cdot \frac{|\eta|(0)-|\eta|(-r)}{\lambda-\alpha} \int_{-r}^{0}\left(\frac{\left\|\phi_{1}(\theta)\right\|_{V}}{\left\|\phi_{0}\right\|_{V}}-\frac{\alpha-\lambda}{|\eta|(0)-|\eta|(-r)}\right)^{2} d|\eta|(\theta) \\
\leq & 0 .
\end{aligned}
$$

Hence, it follows that $\left(\phi,\left(\mathcal{A}+\lambda v^{-1}\right) \phi\right)_{\mathcal{H}} \leq 0$ for all $\phi \in \mathscr{D}(\mathcal{A})$. Due to the equivalence between $\langle\cdot, \cdot\rangle_{\mathcal{H}}$ and $(\cdot, \cdot)_{\mathcal{H}}$, this further implies, in addition to Lemma 2.4, that $C_{0}$-semigroup $e^{t \mathcal{A}}, t \geq 0$, is exponentially stable. The proof is complete now.

\section{Stability of Stochastic Delay Systems}

Let $r>0$ and we consider the following stochastic retarded evolution equation in the real Hilbert space $H$,

$$
\left\{\begin{array}{l}
\mathrm{d} y(t)=A y(t) \mathrm{d} t+A_{1} y(t-r) \mathrm{d} t+\int_{-r}^{0} A_{2}(\theta) y(t+\theta) \mathrm{d} \theta \mathrm{d} t+B y(t) \mathrm{d} W(t), \quad t \geq 0, \\
y(0)=\phi_{0}, \quad y(\theta)=\phi_{1}(\theta), \quad \theta \in[-r, 0], \quad\left(\phi_{0}, \phi_{1}\right) \in \mathcal{H}=H \times L^{2}([-r, 0] ; V),
\end{array}\right.
$$

where $B \in \mathscr{L}\left(H, \mathscr{L}_{2}\left(K_{Q}, H\right)\right), A_{1} \in \mathscr{L}\left(V, V^{*}\right), A_{2}(\cdot) \in L^{2}\left([-r, 0] ; \mathscr{L}\left(V, V^{*}\right)\right)$ and $W(\cdot)$ is a given $Q$-Wiener process.

Let $\mathcal{A}$ be the generator of the delay semigroup $e^{t \mathcal{A}}, t \geq 0$, on $\mathcal{H}=H \times$ $L^{2}([-r, 0], V)$ with

$$
\mathcal{A} \phi=\left(A \phi_{0}+A_{1} \phi_{1}(-r)+\int_{-r}^{0} A_{2}(\theta) \phi_{1}(\theta) \mathrm{d} \theta, \frac{\mathrm{d} \phi_{1}(\theta)}{\mathrm{d} \theta}\right) \quad \text { for any } \phi \in \mathscr{D}(\mathcal{A}) \text {. }
$$

Further, define a linear operator $\mathcal{B} \in \mathscr{L}\left(\mathcal{H}, \mathscr{L}_{2}\left(K_{Q}, \mathcal{H}\right)\right)$ by

$$
\mathcal{B} \phi=\left(B \phi_{0}, 0\right) \quad \text { for any } \quad \phi \in \mathcal{H}
$$

Then, equation (4.1) can be lifted up into an equivalent stochastic evolution equation without delays,

$$
\left\{\begin{array}{l}
\mathrm{d} Y(t)=\mathcal{A} Y(t) \mathrm{d} t+\mathcal{B} Y(t) \mathrm{d} W(t), \quad t \geq 0 \\
Y(0)=\phi, \quad \phi \in \mathcal{H}
\end{array}\right.
$$


where $Y(t)=\left(y(t), y_{t}\right), t \geq 0$. Note that we have the relation $\|\mathcal{B}\|=\|B\|$. Indeed, for any $\phi \in \mathcal{H}$, it is easy to see that

$$
\|\mathcal{B} \phi\|_{\mathcal{H}}^{2}=\left\|\left(B \phi_{0}, 0\right)\right\|_{\mathcal{H}}^{2}=\left\|B \phi_{0}\right\|_{H}^{2} \leq\|B\|^{2}\left\|\phi_{0}\right\|_{H}^{2} \leq\|B\|^{2}\|\phi\|_{\mathcal{H}}^{2},
$$

thus, $\|\mathcal{B}\| \leq\|B\|$. On the other hand, let $\phi=\left(\phi_{0}, 0\right), \phi_{0} \in H$, then it follows that

$$
\left\|B \phi_{0}\right\|_{H}^{2}=\|\mathcal{B} \phi\|_{\mathcal{H}}^{2} \leq\|\mathcal{B}\|^{2}\|\phi\|_{\mathcal{H}}^{2}=\|\mathcal{B}\|^{2}\left\|\phi_{0}\right\|_{H}^{2},
$$

which immediately implies that $\|B\| \leq\|\mathcal{B}\|$. Hence, we finally obtain the equality $\|B\|=\|\mathcal{B}\|$.

Now let $K=\mathbb{R}, W(t)=w(t)$, a standard real Brownian motion, $A_{2}(\cdot) \equiv 0$, $B \in \mathscr{L}(H)$, then we have for any $\beta \in \mathbb{R}$ that

$$
(\mathcal{A}+\beta \mathcal{B}) \phi=\left((A+\beta B) \phi_{0}+A_{1} \phi_{1}(-r), \frac{\mathrm{d} \phi_{1}(\theta)}{\mathrm{d} \theta}\right) .
$$

Hence, by virtue of Proposition 2.1 and Theorem 3.2, we have that if there exists a number $\beta \in \mathbb{R}$ such that $A+\beta B$ generates an exponentially stable $C_{0}$-semigroup and

$$
\left\|e^{t(A+\beta B)}\right\| \leq M e^{-\alpha t}, \quad \frac{\beta^{2}}{2}+\|B\|^{2} \leq 2 \lambda,\left\|A_{1}\right\|^{2} e^{2 \lambda r}<(\alpha-\lambda)^{2} \text { for some } \alpha>\lambda>0
$$

then the mild solution $Y$ of (4.3) (thus, $y$ of (4.1)) is exponentially stable in the almost sure sense. In this case, if $A_{1} \neq 0$, the delay parameter $r>0$ satisfies

$$
r<\frac{1}{\lambda} \ln \left(\frac{\alpha-\lambda}{\left\|A_{1}\right\|}\right)
$$

Last, let us study an example in which the delay appears in the highest-order derivative term of the stochastic system to close this work. The example (1.10) in Section 1 can be also treated in a similar manner.

Example 4.1 First consider the following linear stochastic partial differential equation,

$$
\left\{\begin{array}{l}
\mathrm{d} y(t, \xi)=\left(1+\alpha_{0}\right) \frac{\partial^{2}}{\partial \xi^{2}} y(t, \xi) \mathrm{d} t+\sigma y(t, \xi) \mathrm{d} w(t), \quad t \geq 0, \quad \xi \in[0, \pi] \\
y(t, 0)=y(t, \pi)=0, \quad t \geq 0 \\
y(0, \cdot)=\phi_{0}(\cdot) \in L^{2}(0, \pi)
\end{array}\right.
$$

where $\alpha_{0} \in \mathbb{R}$ and $w$ is a standard real Brownian motion. By virtue of Example 2.1, it is known that if

$$
-1-\alpha_{0}<\sigma^{2} / 2
$$


the null solution of (4.6) is exponentially stable in the almost sure sense.

Now let $r>0$ and consider the following linear stochastic delay partial differential equation,

$$
\left\{\begin{array}{l}
\mathrm{d} y(t, \xi)=\frac{\partial^{2}}{\partial \xi^{2}} y(t, \xi) \mathrm{d} t+\alpha_{0} \frac{\partial^{2}}{\partial \xi^{2}} y(t-r, \xi) \mathrm{d} t+\sigma y(t, \xi) \mathrm{d} w(t), \quad t \geq 0, \quad \xi \in[0, \pi] \\
y(t, 0)=y(t, \pi)=0, \quad t \geq 0 \\
y(0, \cdot)=\phi_{0}(\cdot) \in L^{2}(0, \pi), \quad y_{0}(\cdot, \cdot)=\phi_{1}(\cdot, \cdot) \in L^{2}\left([-r, 0] ; L^{2}(0, \pi)\right)
\end{array}\right.
$$

We show that under the condition $-1-\alpha_{0}<\sigma^{2} / 2$, the pathwise exponential stability of the null solution of (4.7) is not sensitive to small delays $r>0$.

Indeed, let $V=H_{0}^{1}(0, \pi) \cap H^{2}(0, \pi), H=L^{2}(0, \pi)$ and $\mathcal{A}$ be the generator of the associated delay semigroup $e^{t \mathcal{A}}, t \geq 0$, in $\mathcal{H}=H \times L^{2}([-r, 0], V)$ with

$$
\mathcal{A} \phi=\left(\Delta \phi_{0}+\alpha_{0} \Delta \phi_{1}(-r), \mathrm{d} \phi_{1}(\theta) / \mathrm{d} \theta\right) \quad \text { for } \phi=\left(\phi_{0}, \phi_{1}\right) \in \mathscr{D}(\mathcal{A}),
$$

where $\Delta=\partial^{2} / \partial \xi^{2}$ is the standard Laplace operator. Once again, let $\mathcal{B} \in \mathscr{L}(\mathcal{H})$ be given by $\mathcal{B} \phi=\left(\sigma \phi_{0}, 0\right)$ for $\phi=\left(\phi_{0}, \phi_{1}\right) \in \mathcal{H}$. Then,

$$
(\mathcal{A}+\beta \mathcal{B}) \phi=\left(\Delta \phi_{0}+\alpha_{0} \Delta \phi_{1}(-r)+\beta \sigma \phi_{0}, \mathrm{~d} \phi_{1}(\theta) / \mathrm{d} \theta\right), \quad \phi \in \mathscr{D}(\mathcal{A}) .
$$

By virtue of (4.4), we have that if there exists some number $\beta \in \mathbb{R}$ such that for some $\lambda>0$,

$$
\lambda<1-\beta \sigma, \quad \frac{\beta^{2}}{2}+\sigma^{2} \leq 2 \lambda, \quad \alpha_{0}^{2} e^{2 \lambda r}<(1-\beta \sigma-\lambda)^{2},
$$

then the null solution of (4.7) is exponentially stable in the almost sure sense. It may be verified that $\beta=-2 \sigma$ and $\lambda=\frac{3}{2} \sigma^{2}+\varepsilon$ with $\varepsilon>0$ sufficiently small satisfy condition (4.8). In this case, the third inequality in (4.8) is actually reduced to

$$
\left|\alpha_{0}\right| \exp \left(3 \sigma^{2} r / 2\right)-1<\sigma^{2} / 2
$$

If $\alpha_{0} \neq 0, \sigma \neq 0$, this condition is equivalent to

$$
r<\frac{2}{3 \sigma^{2}} \ln \left(\frac{\frac{1}{2} \sigma^{2}+1}{\left|\alpha_{0}\right|}\right)
$$

In other words, in addition to the condition $-1-\alpha_{0}<\sigma^{2} / 2$ which ensures the pathwise exponential stability of Eq. (4.6), if we further assume that the relation (4.9) is true, then the null solution of (4.7) is exponentially stable in almost sure sense. Thus, the stability is not sensitive to small delays. 
Open Access This article is distributed under the terms of the Creative Commons Attribution 4.0 International License (http://creativecommons.org/licenses/by/4.0/), which permits unrestricted use, distribution, and reproduction in any medium, provided you give appropriate credit to the original author(s) and the source, provide a link to the Creative Commons license, and indicate if changes were made.

\section{References}

1. Appleby, J.A., Mao, X.R.: Stochastic stabilisation of functional differential equations. Syst. Control Lett. 54(11), 1069-1081 (2005)

2. Bátkai, A., Piazzera, S.: Semigroups for Delay Equations. Research Notes in Math., A.K. Peters, Wellesley, Massachusetts, (2005)

3. Bierkens, J.: Pathwise stability of degenerate stochastic evolutions. Integral Equ. Oper. Theory 23, $1-27(2010)$

4. Da Prato, G., Zabczyk, J.: Stochastic Equations in Infinite Dimensions. Encyclopedia of Mathematics and its Applications, 2nd edn. Cambridge University Press, Cambridge (2014)

5. Datko, R.: Not all feedback stabilized systems are robust with respect to small time delays in their feedback. SIAM J. Control Optim. 26, 697-713 (1988)

6. Datko, R., Lagnese, J., Polis, M.: An example on the effect of time delays in boundary feedback of wave equations. SIAM J. Control Optim. 24, 152-156 (1986)

7. Di Blasio, G., Kunisch, K., Sinestrari, E.: $L^{2}$-regularity for parabolic partial integrodifferential equations with delay in the highest-order derivatives. J. Math. Anal. Appl. 102, 38-57 (1984)

8. Jeong, J.-M., Nakagiri, S., Tanabe, H.: Structural operators and semigroups associated with functional differential equations in Hilbert spaces. Osaka J. Math. 30, 365-395 (1993)

9. Liptser, R.: A strong law of large numbers for local martingales. Stochastics 3, 217-228 (1980)

10. Liu, K.: Stability of Infinite Dimensional Stochastic Differential Equations with Applications. Chapman \& Hall/CRC, Taylor \& Francis, New York, London, (2006)

11. Mao, X.R.: Stochastic stabilization and destabilization. Syst. Control Lett. 23, 279-290 (1994)

12. Mao, X.R.: Stochastic Differential Equations and Applications, 2nd edn. Woodhead Publishing, Oxford (2007)

13. Revuz, D., Yor, M.: Continuous Martingales and Brownian Motion, 3rd edn. Springer, Berlin (1999)

14. Smith, H.: An Introduction to Delay Differential Equations with Applications to the Life Sciences. Springer, Berlin (2011) 